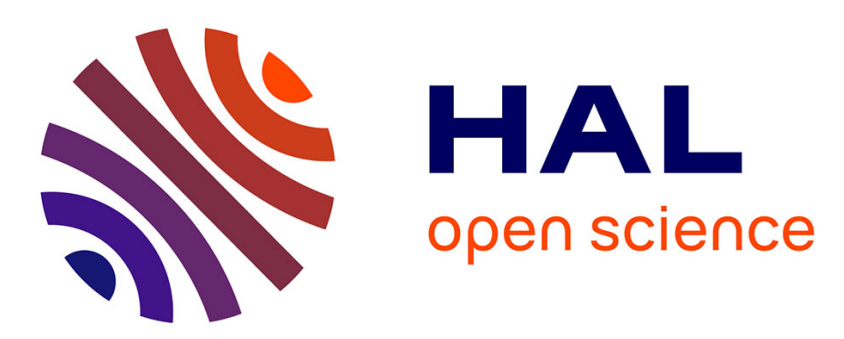

\title{
Influence of the grid cell geometry on 3D cellular automata behavior in intergranular corrosion
}

Simone Guiso, Dung Di Caprio, Jacques de Lamare, Benoît Gwinner

\section{To cite this version:}

Simone Guiso, Dung Di Caprio, Jacques de Lamare, Benoît Gwinner. Influence of the grid cell geometry on 3D cellular automata behavior in intergranular corrosion. Journal of computational science, 2021, 53, pp.101322. 10.1016/j.jocs.2021.101322 . hal-03261073

\section{HAL Id: hal-03261073 \\ https://hal.science/hal-03261073}

Submitted on 17 Nov 2021

HAL is a multi-disciplinary open access archive for the deposit and dissemination of scientific research documents, whether they are published or not. The documents may come from teaching and research institutions in France or abroad, or from public or private research centers.
L'archive ouverte pluridisciplinaire HAL, est destinée au dépôt et à la diffusion de documents scientifiques de niveau recherche, publiés ou non, émanant des établissements d'enseignement et de recherche français ou étrangers, des laboratoires publics ou privés. 


\title{
Influence of the grid cell geometry on 3D cellular automata behavior in intergranular corrosion
}

\author{
Simone Guiso ${ }^{1,2, *}$, Dung di Caprio ${ }^{2}$, Jacques de Lamare ${ }^{1}$, Benoît Gwinner ${ }^{1}$ \\ *simone.guiso@cea.fr \\ ${ }^{1}$ Den-Service de la Corrosion et du Comportement des Matériaux dans leur Environnement \\ (SCCME), CEA, Université Paris-Saclay, F-91191, Gif-sur-Yvette, France \\ ${ }^{2}$ Chimie ParisTech, PSL Research University, CNRS, Institut de Recherche de Chimie Paris \\ (IRCP), F-75005 Paris, France
}

\begin{abstract}
The bulk structure of stainless steels (SS) consists of mostly convex grains whose thin (one $\mathrm{nm}$ ) planar interfaces are called grain boundaries (GB). There are industrial contexts where SS exposed to aggressive oxidative media undergo a fast degradation due to inter-granular corrosion (IGC), a preferential attack that occurs at the grain boundaries, propagating along them and leading to grains detachment. To address the IGC issue, we developed a 3D synchronous cellular automata (CA) model. Our first goal was to obtain a GB corrosion rate that could be as isotropic as possible. We performed full angular studies, with respect to the spatial orientation of a single grain boundary, in three different cases of grid (array of cells) geometry (cubic, hexagonal close-packed (HCP), face-centered cubic (FCC)). The HCP grid appears to be the best choice, as far as isotropy of the GB corrosion rate is concerned.
\end{abstract}

Keywords: cellular automata, intergranular corrosion, modelling, lattice comparison 


\section{Introduction}

Corrosion phenomena have a considerable impact on worldwide industrial processes. They always involve a combination of a material and an environment. In the case of stainless steels (SS) exposed to aggressive oxidative media, as it could happen for example in the industry of nuclear spent fuel reprocessing, one of the observed corrosion phenomena can be the intergranular corrosion (IGC) [1-3]. A macroscopic and schematic view of a SS sample in contact with an aggressive liquid is represented in Fig. 1. In the real life, the granular structure of SS is most often made of grains that are mostly convex, due to their (spatially uniform but not necessarily isotropic) growth mechanism from randomly distributed seeds. The size distributions of these grains exhibit first moments that may vary on several orders of magnitude (from $\mu \mathrm{m}$ to $\mathrm{mm}$, depending on the particular material being considered), while the thickness of their planar interfaces is around one $\mathrm{nm}$. In this paper, we focus on the IGC phenomenon, in which the corrosion of the grain boundaries (GB) (in red in Fig. 1) is greatly faster than the dissolution of the grains themselves. We consider the most common case where, in a given SS macroscopic sample with a large number of grains (like in Fig. 1), GB have no preferential spatial orientation. It means that even if there are different physicochemical classes of GB (mainly due to particular cases of crystallographic misorientations between adjacent grains [4]), each class is enough populated in the sample and spatially randomly oriented, so that you can consider a global isotropy of the GB normal vectors distribution. Then, from a statistic point of view, GB corrosion velocities must also appear to be globally isotropic (that is, independent of the planar GB spatial orientation). Indeed, that is what is experimentally observed at a macroscopic (statistic) scale. That is why we decided, in order to properly simulate IGC, to build a cellular automata (CA) model that gives $\mathrm{GB}$ corrosion rates that are as isotropic as possible. If we could obtain a purely isotropic GB corrosion rate with such a CA model, we would a get a numerical tool that could be useful for industrial applications. Indeed it could serve to the search of the minimum paths lengths (which are key parameters to estimate the life duration of devices suffering IGC) through the 3D network of intersecting planar facets of randomly distributed convex grains, and related statistical properties. Of course, the fact that we use CA modelling means that we would tackle the discrete and not continuous version of the problem, which resists to mathematicians until today. 


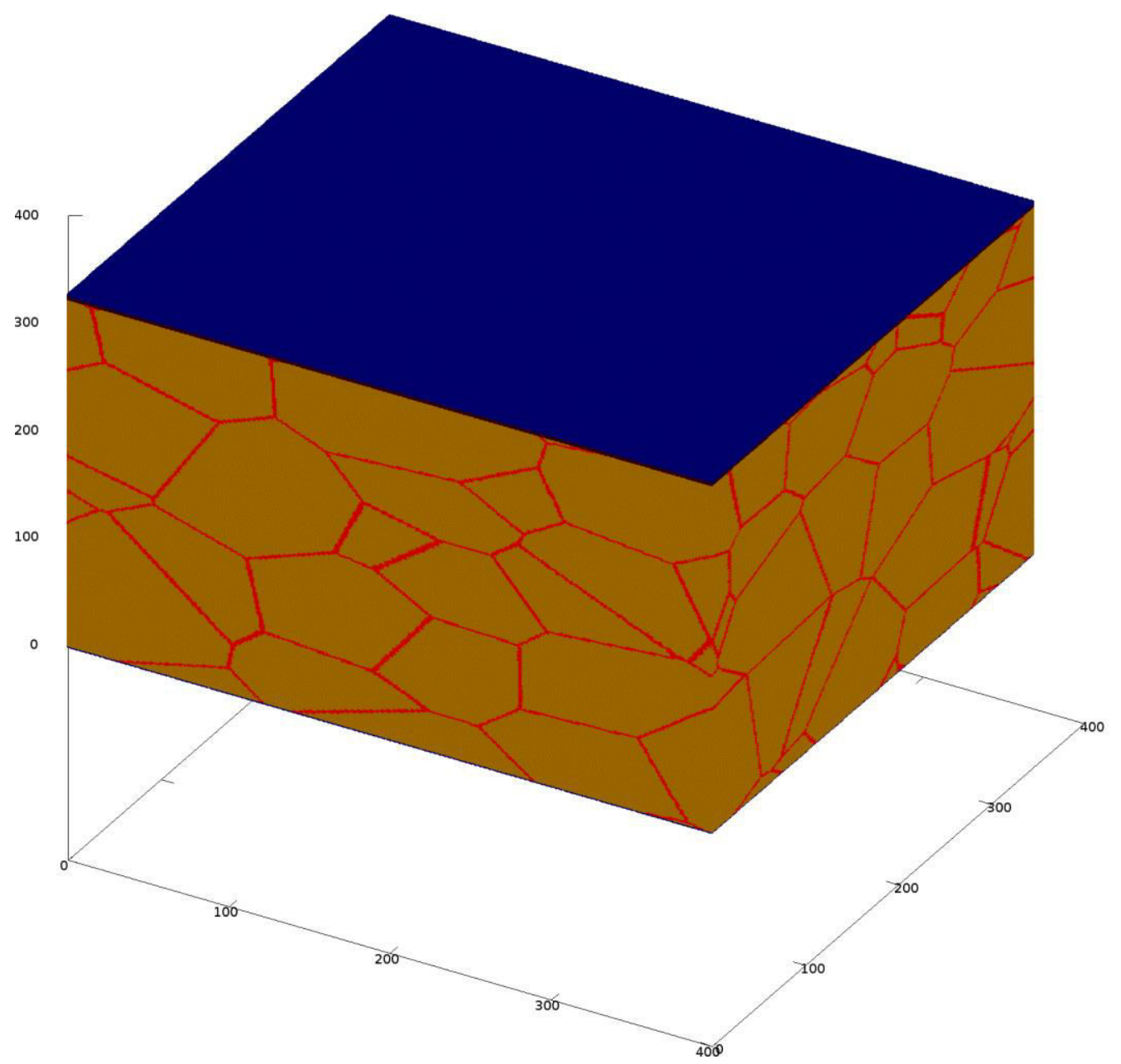

Figure 1 - Schematic 3D representation of the granular structure of a SS sample in contact with a thin layer of aggressive liquid media (in blue). Grains are in brown, GB in red. On-axis indexes represent pixel numbers, just for the figure.

IGC modelling and related numerical simulations as well can be found elsewhere in the literature, involving different computational methods.

Lim et al. [5] performed Monte-Carlo simulations to estimate time-dependent IGC damage depth progression in Al-Mg alloys: a Voronoï diagram was constructed to model a pristine granular material, where only the top surface was exposed to the corrosion agent. Monte-Carlo simulations were also compared to a stochastic reduced order model (SROM) by Sarkar et al. [6] to quantify the uncertainty of intergranular corrosion rates. Results showed that SROMs are more computationally efficient than Monte-Carlo simulations and then constitute an accurate alternative for this purpose. Other models focused on the material microstructure, having as input parameters the geometries and grain orientation[7-8].

A quite promising and recent approach is to model corrosion phenomena with cellular automata (CA) methods. Bartosik et al. [9] successfully obtained a 3D model off a two-steps anodization process 
matching theoretical predictions, while Perez-Brokate et al. [10] and Van der Weeen et al. [11] focused their studies on pitting corrosion. Other CA corrosion studies can be found in [12-16].

Jahns et al. [17] developed a 2D CA model to study IGC, focusing their attention on the description of diffusing paths at grain boundary level. The model was applied to two experimental cases, where the comparison between simulations and experimental results showed a good agreement.

Di Caprio et al. [18] generalised previous 2D numerical models [19] by simulating the effect of grain size on the IGC in a 3D probabilistic CA structure. Ueno et al. [20-21] also developed a 3D cellular automata model to study the intergranular corrosion and fracture in SS. Different corrosion probabilities were taken into account in their model, each of them depending on species concentration at GB. A probabilistic 3D model was also developed by Lishchuk et al. [22] who extended their own "brick-wall" model [23] by extracting the material microstructure from 3D electron backscattered diffraction (EBSD) experiments. A differential evolution algorithm was then used to fit the parameters of the CA model with experimental data. Simulations of the progression of the corrosion front showed a good agreement with experimental data.

All these models brought significant results in terms of accuracy by always getting closer to real cases. For evident "simplicity of numerical implementation" reasons however, the corrosion CA models are most often based on a cubic grid. In the particular case of IGC modelling by CA, we decided to tackle the central question of the dependence of the isotropicity properties of the GB corrosion rate on the grid geometry. In order to get quantitative results, we performed a full angular screening of the corrosion velocities of planar GB as a function of their spatial orientations, with three different types of grids: a cubic grid, a hexagonal closed-packed (HCP) grid and a face-centred cubic (FCC) grid (in the case of FCC-111 orientation). Let us mention that in other engineering applications [24], hexagonal close-packed grids have been used, with good results.

The paper is organized as follows. We describe the CA approach in Section 2. The simulation setup for the different studies is presented in Section 3, while results and discussion are given in Section 4.

\section{The cellular automata approach}

Cellular automata, originated and firstly theorized by Von Neumann [25], are discrete and dynamical systems that consist of a regular network of finite state automata (named cells) that change their state depending on the states of their neighbours, according to a local update (or transition) rule which is uniform over the network. The system dynamics is investigated through iterations of the updating process. Then it requires some "external links" to correlate the iteration number to a real time evolution, if needed. In the case where each cell is updated at every iteration the CA is called synchronous, if not, it is called asynchronous. There is a huge literature about CA applications, which have covered almost all 
fields of academic and industrial research for many decades, like (non-exhaustive list): biology, epidemiology, human and social sciences, ecology, fluid dynamics, geomorphology, fast computing sciences, nanomaterials. An overview of these applications would be far beyond the scope of the present paper.

For this specific IGC study we use a very simple synchronous CA model, which is focused exclusively on GB corrosion and whose definition rests upon the four following points:

- Grid: The domain is firstly divided into a 3D array of identical cells whose co-ordinates are listed in a Cartesian reference system. This array is called a grid.

- List of states: At each iteration, there are three possible states for a given cell. If a cell is part of the material structure, it can be "grain" (GN), or "inter-granular" (IGN) (that is, representing GB). If it is part of the corrosive liquid medium, its state is "solution" (SOL).

- Neighbourhood: It corresponds to the list of neighbouring cells of a given one. The definition of this neighbourhood has to be uniform throughout the grid. Note that the number and spatial arrangement of these neighbouring cells strongly depend on the grid that is used and then play an important role concerning the isotropicity properties we investigate in this paper. Detailed explanations are given below.

- Transition rules: The rules that govern the dynamics of the system are the following. A cell that is SOL at iteration i cannot evolve further and will remain SOL. A cell which is GN initially (that is, at iteration $\mathrm{i}=0$ ) does not evolve, it stays GN (corrosion does not affect grains themselves). A cell which is IGN at iteration i evolves to SOL state at iteration i+1 if and only if there is at least one SOL cell in its neighbourhood at iteration $i$ (which describes the corrosion of GB in terms of dissolution).

In this study the type of grid can be considered as a parameter. We set the distance between a given cell and its nearest neighbour, whatever the grid, equal to 1 . We present the three (cubic, HCP, FCC) type of grids that we investigated in the following figures (respectively Fig. 3, 4 and 5). The cells are represented as spheres, but let us note that this has no particular physical meaning, we have chosen to use spheres (that is discs in the views from above) in the sole concern to make the figures as readable as possible. Only the Cartesian coordinates of these spheres centres matter and characterize the different types of grids. The distances we discuss here have to be considered as distances between cells centres. A schematic representation of what is called a 3D "Moore neighbourhood" in the CA literature in the case of a cubic grid is given in Fig.2. It means that a given cell (in red) has 26 neighbouring cells: 6 face-cells at distance of 1 (in blue), 12 edge-cells at distance of $\sqrt{2}$ (in green) and 8 corner-cells at distance of $\sqrt{3}$ (in black). Note that for the cubic grid the $z$ axis is a rotation symmetry axis of order 4 . 


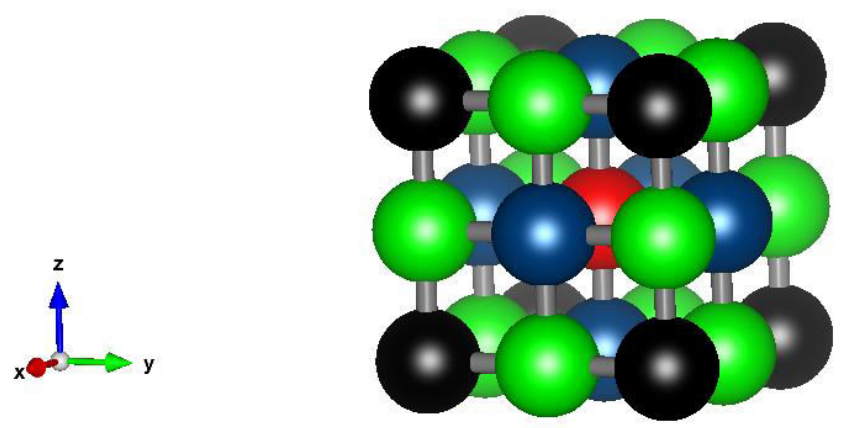

Figure 2: Schematic view of the 3D “Moore" neighborhood (blue, green and black spheres) of a given cell (in red) in the case of a cubic grid.

A schematic view from above of the spatial arrangement of cells in the HCP grid is represented in Fig.3. It can be viewed as the stacking in the $z$ direction (perpendicular to the figure) of planar arrangements that are just shifted from one plane to the other. In the HCP case the stacking type is ABA, which means that the structure $A B$ is repeated with a periodicity of " 2 planes" in the $z$ direction. This spatial arrangement corresponds to a solution of maximum density for hard spheres stacking (see Kepler conjecture). A given cell is surrounded by 12 equally spaced cells: 6 cells on the same z-plane, 3 cells in the overlying plane and 3 cells in the plane below (in matching positions with respect to the 3 cells of the underlying plane). Note that, with this type of grid, the space could be completely filled using identical dodecahedral cells. Both the equality of the distance between adjacent cells and the fact that the $z$ axis is an axis of symmetry of order 6 are properties that make the use of the HCP grid promising in our CA model for IGC, in terms of isotropicity of the corrosion rates properties. Finally, one can note that the space sampling associated to the HCP neighbourhood is lower than that of the cubic grid (12 neighbours instead of 26).

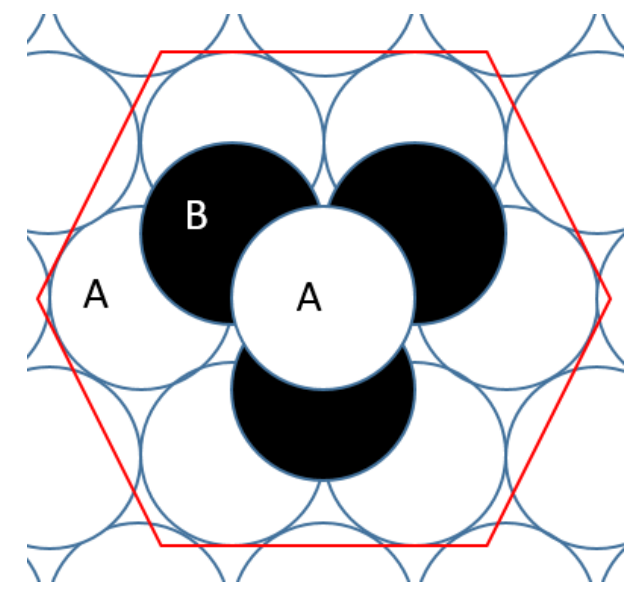

Figure 3: Schematic view from above of the spatial arrangement of cells in the case of the HCP grid, that is obtained by periodic stacking of the A (in white) and B (in black) sequence of planar structures in the $z$ direction (perpendicular to the figure). A and B planar arrangements are identical and just shifted from one another, along the plane of the figure. 
FCC-grid has the same crystal system as the cubic grid [2] and presents some similarities with the HCP grid, as the same high packaging factor in the case of identical spherical cells ( $74 \%)$. A schematic representation of the FCC-111 stacking is given in Fig.4. One can see that it is very similar to the HCP stacking, but the pattern is a bit different, since the FCC grid is obtained by periodically repeating the $A B C$ stacking sequence in the $z$ direction. As in the case of HCP a given cell is surrounded by 12 equally spaced cells, the planar structures $A, B$ and $C$ are identical and just shifted from one another, but here the $z$ periodicity of the planar stacking sequence is 3 (instead of 2 for HCP grid). Let us finally note that the $z$ axis is an axis of symmetry of order 3 in the case of FCC grid.

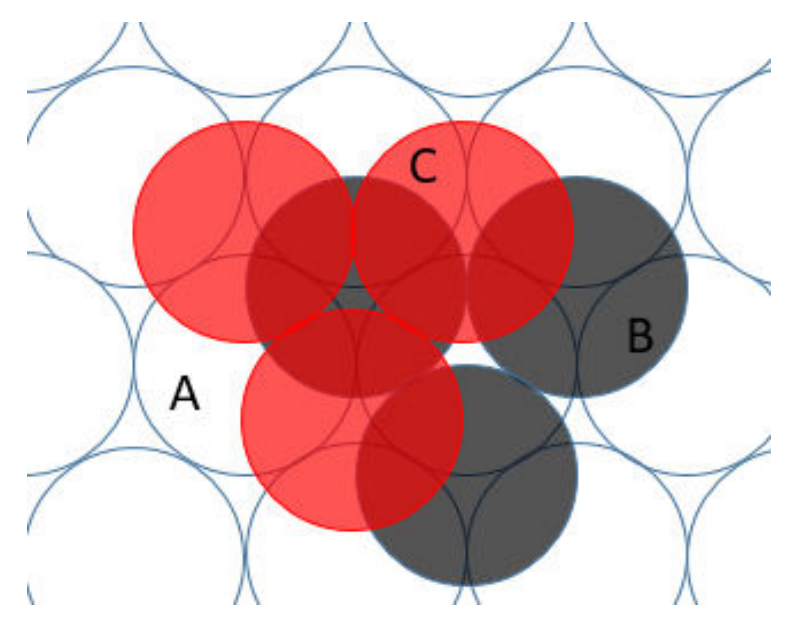

Figure 4: Schematic view from above of the spatial arrangement of cells in the case of the FCC grid, that is obtained by periodic stacking of the A (in white), B (in grey) and C (in red) sequence of planar structures in the $z$ direction (perpendicular to the figure). A, B and C planar arrangements are identical and just shifted from one another, along the plane of the figure.

This CA model has been implemented in CUDA environment for parallel processing on Graphics Processing Units (GPU).

\section{Simulation setup}

To characterize a planar GB, we use the polar and azimuthal angles $(\theta, \varphi)$ of its normal, as represented in Fig.5. The planar $\mathrm{GB}$ is the one that contains the geometrical centre $\mathrm{O}\left(x_{0}, y_{0}, z_{0}\right)$ of the grid. 


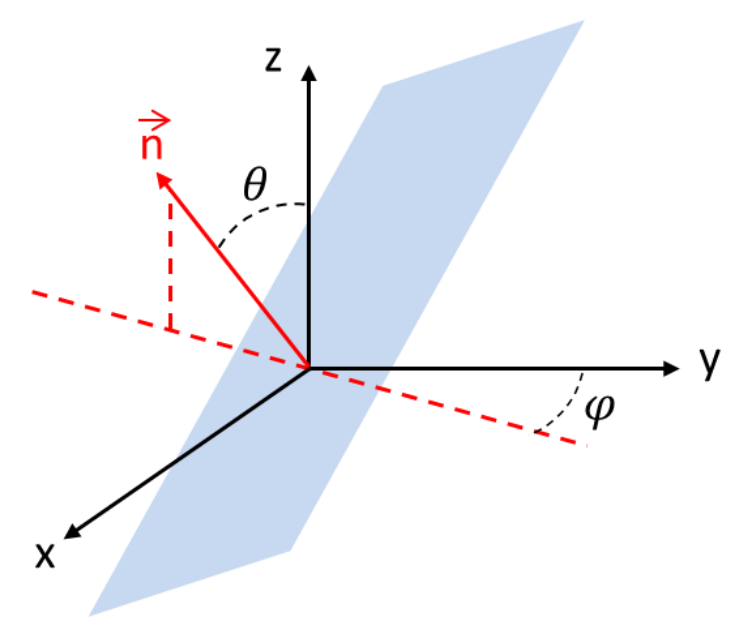

Figure 5 - Schematic 3D view of a planar GB (in blue) and notations used to characterized it by the polar and azimuthal angles $(\theta, \varphi)$ of its normal $\vec{n}$ (in red). When $\theta$ is equal to 0 (the normal $\vec{n}$ is coincident with the $z$ vector), the plane is horizontal.

Simulations were performed on $(\mathrm{Nx}=2048, \mathrm{Ny}=2048, \mathrm{Nz}=256)$ grids, whatever their (cubic, HCP or FCC111) type, which therefore contain around $10^{9}$ cells. In the initial state of the grid, the two uppermost $(z=$ $\mathrm{Nz}-1$ and $\mathrm{z}=\mathrm{Nz}$ ) layers consist of SOL cells, so that the corrosion progresses downwards, in the direction of decreasing z. A side view of an elementary IGC process is represented in Fig. 6, as an example, in the case of a single $\left(\theta=60^{\circ}, \varphi=0\right) \mathrm{GB}$ on a cubic grid. IGN red cells represent the GB and GN brown cells represent the adjacent grains. We clearly see the evolution of the corrosion front during the IGC process (progressive dissolution of the GB), where initially IGN cells become SOL cells (in white).

(a)

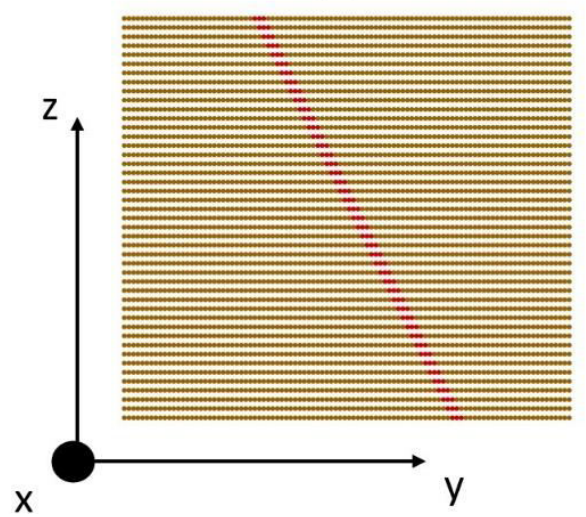

(b)

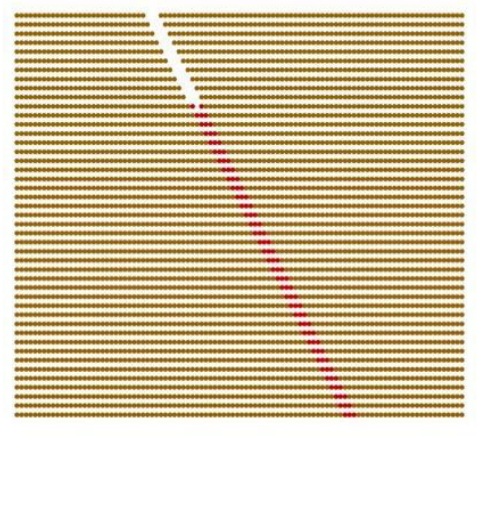

(c)

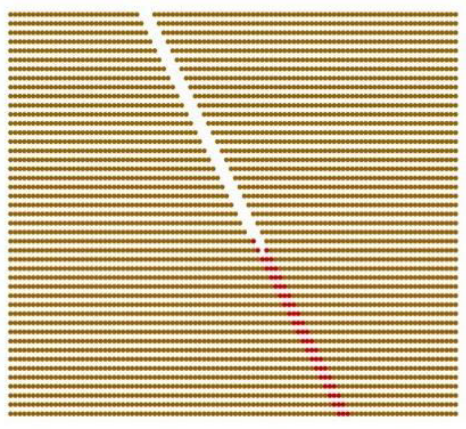

Figure 6 - Side view of the evolution of IGC corrosion in the case of a $\left(\theta=60^{\circ}, \varphi=0\right)$ GB (in red). GN cells are in brown, and SOL cells in white. (a) initial state, where the two uppermost layers does not appear since they consist of white SOL cells. (b) at iteration $i=20$. (c) at iteration $i=40$. The average thickness of the grain boundary region is equal to 4 .

To initialize the states of all the cells of the grid in a particular $(\theta, \varphi) \mathrm{GB}$ case, special care has to be taken concerning the calculation that has to be made to determine whether a given cell of the grid is initially a IGN cell or not. To do that, the first step is to fix the grain boundary thickness value $e_{G B}$, which is expressed as a multiple of the unit distance. Then we set two criterias. The first one involves the 
calculation of the euclidian distance between the given cell and the $(\theta, \varphi)$ plane under consideration. If this distance is more than half the thickness of the grain boundary, then the cell is not initially IGN.

The second criteria is set in order to avoid boundary effects. Indeed, the intersection between a given $(\theta, \varphi)$ plane and a given horizontal (constant $z$ ) plane is a line, whose restriction to the parallelepiped shaped volume of the grid is a line segment, whose length can be written as $L(\theta, \varphi, z)$, as shown in Fig. $7(a)$. Then, the second geometrical criteria just consists in setting lateral restrictions to the initial GB volume, so that $L(\theta, \varphi, z)$ no more depends on $z$, that is $L(\theta, \varphi, z)=L(\theta, \varphi)$. In other words the $G B$ is cut laterally by vertical planes, if needed. Initial IGN cells are those which meet both criterias. Note that while applying these two criteria, we ensure that the intersection of any $e_{G B}$ thick $(\theta, \varphi) G B$ with any given "constant $z^{\prime}$ (horizontal) plane is a rectangle whose area $A_{G B}(\theta, \varphi)$ is the product $\left(e_{G B} / \sin \theta\right) * L(\theta, \varphi)$ (see Fig. 7(a) and (b)), that does not depend on z. As a consequence, we also see that the initial number of IGN cells in a given $z$ plane of the grid does not much depend on $z$, except due to small discretization effects that generate some roughness and which we cannot avoid: we call it $N A_{G B}(\theta, \varphi)$.

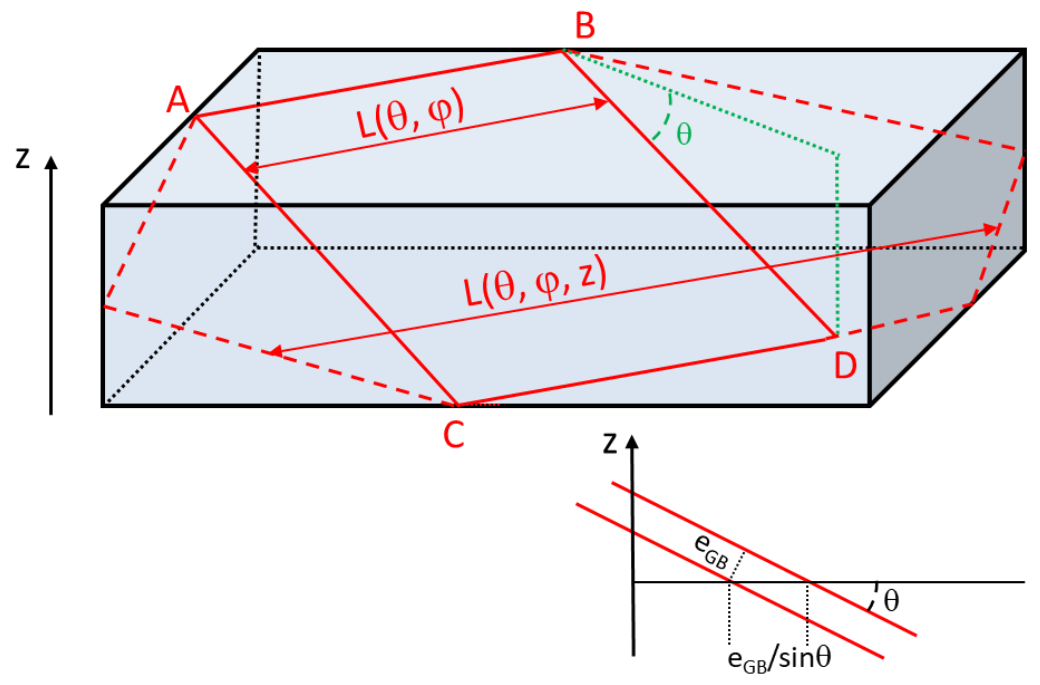

Figure 7 - (a) Schematic representation of the polygonal intersection (red dashed line) between a given $(\theta, \varphi)$ plane and a parallelepiped shaped box (volume of the grid). We set the second criteria to restrict this intersection to a rectangle (ABCD red solid line), so that $L(\theta, \varphi, z)=L(\theta, \varphi)$ throughout a given $(\theta, \varphi)$ simulation. (b) Side view of the intersection of a $e_{G B}$ thick $(\theta, \varphi)$ GB (in red) with a given $z=$ constant plane.

Let us remark that $\mathrm{NA}_{G B}(\theta, \varphi)=\mathrm{N}_{\mathrm{IGN}}(\mathrm{i}=0) /(\mathrm{Nz}-2)$, that is the ratio between the initial (at iteration $\mathrm{i}=0$ ) total number of IGN cells in the grid and the total number of "material state" planes, which is $\mathrm{Nz}-2$ since the 2 uppermost layers of the grid are made of SOL cells. As we explained in the introduction, we want to fully investigate the comparison between the CA simulated GB corrosion rate and the theoretical isotropic value, as a function of $(\theta, \varphi)$ angles. To do this, we first defined a dimensionless z-component of the simulated corrosion rate $v_{z, \text { sim }}(\mathrm{i})$ at the $\mathrm{i}$-th iteration as, for a given $(\theta, \varphi)$

$v_{z, \operatorname{sim}}(i)=\frac{N_{I G N}(i-1)-N_{I G N}(i)}{N A_{G B}(\theta, \varphi)} * A_{\text {grid }}$ 
that is the ratio between the total number of IGN cells that disappeared at the i-th iteration $\left(N_{I G N}(i-1)-\right.$ $\left.\mathrm{N}_{\mathrm{IGN}}(\mathrm{i})\right)$ and the number $\mathrm{NA}_{\mathrm{GB}}(\theta, \varphi)$ of IGN cells initially present in each z plane of the grid, multiplied by a purely geometrical "grid factor" $A_{\text {grid }}$ which was set to take into account the grid dependency of the distance between two consecutive z-planes of the grid. The values of $A_{\text {grid }}$ are given in the following table:

Table 1 - Geometrical distance $\boldsymbol{A}_{\text {grid }}$ between two consecutive z-planes

\begin{tabular}{|c|c|c|}
\hline Cubic grid & HCP grid & FCC grid \\
\hline 1 & $\frac{\sqrt{6}}{3}$ & $\frac{\sqrt{6}}{3}$ \\
\hline
\end{tabular}

Let us point out that by calculating $v_{z, \operatorname{sim}}(i)$ with the definition (1), we get answers to the question "what is the fraction of a $z=$ constant slice of the discretized GB which is corroded during the i-th iteration ?" (the answer is $\left.\left(\mathrm{N}_{\mathrm{IGN}}(\mathrm{i}-1)-\mathrm{N}_{\mathrm{IGN}}(\mathrm{i})\right) / \mathrm{NA}_{\mathrm{GB}}(\theta, \varphi)\right)$ and to the question "then what is the equivalent zthickness of the slice of the GB that has been corroded during the i-th iteration ?" (the answer is "that is exactly what we call $\left.v_{z, \operatorname{sim}}(i)^{\prime \prime}\right)$.

Then, we calculated the mean of $v_{z, \operatorname{sim}}(i)$ over $\mathrm{i}\left(\mathrm{i}=1, \ldots, i_{\text {max }}\right.$, where $i_{\text {max }}$ is the total number of iterations of a given simulation). In what follows we call $v_{z, \operatorname{sim}}(\theta, \varphi)$ this mean.

Our main purpose is to compare $v_{z, \operatorname{sim}}(\theta, \varphi)$ to the value $v_{z, t h}$ of the z-component of the GB normalized corrosion rate, $v_{i g n}$, that would correspond to a purely isotropic IGC phenomenon (the GB corrosion rates are the same in any possible direction inside a given GB, in a uniform way inside the GB and whatever the $\mathrm{GB}(\theta, \varphi)$ angles). By supposing that $v_{i g n}=1$ (normalization), $v_{z, t h}$ is simply given by $v_{z, t h}=\sin \theta$, as shown in Fig. 8, so that we have to compare $v_{z, \operatorname{sim}}(\theta, \varphi)$ to $\sin \theta$ for every $(\theta, \varphi)$ angle.

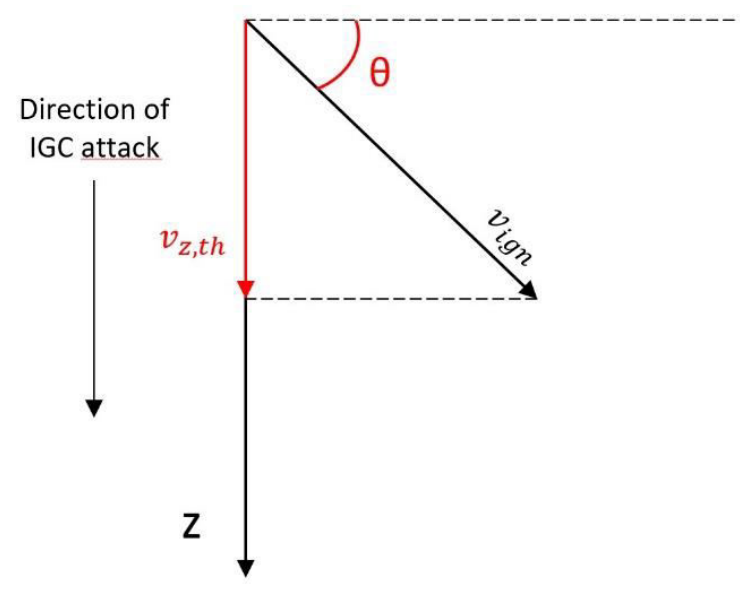

Figure 8 - Schematic representation of the definition $\mathrm{v}_{\mathrm{z}, \mathrm{th}}=\sin \theta$

To obtain a complete description of the behavior of a single grid, we achieved full $(\theta, \varphi)$ screenings, each 5 degrees for both $\left.\theta \in] 0,90^{\circ}\right]$ and $\varphi \in\left[0,360^{\circ}\right.$. We also investigated the sensitivity of the results to the 
GB thickness $\mathrm{e}_{\mathrm{GB}}$. It appears that it does not influence $v_{z, \operatorname{sim}}(\theta, \varphi)$ in a significant way, whatever $(\theta, \varphi)$ and the grid. Results for $(\theta, \varphi=0)$ and HCP grid are presented as examples in Table 2 .

Table 2 - Influence of the GB thickness $\mathrm{e}_{\mathrm{GB}}$ on the calculated GB corrosion rate, HCP grid, $\varphi=0$

\begin{tabular}{|c|c|c|}
\hline$\theta\left[{ }^{\circ}\right]$ & $\mathrm{e}_{\mathrm{GB}}$ (in cells) & $v_{z, \operatorname{sim}}(\theta, \varphi=0)$ \\
\hline 30 & 2 & 0.52 \\
\hline 30 & 4 & 0.52 \\
\hline 30 & 6 & 0.52 \\
\hline 30 & 8 & 0.52 \\
\hline 45 & 2 & 0.76 \\
\hline 45 & 4 & 0.76 \\
\hline 45 & 6 & 0.76 \\
\hline 45 & 8 & 0.76 \\
\hline 60 & 2 & 1.00 \\
\hline 60 & 4 & 1.00 \\
\hline 60 & 6 & 1.00 \\
\hline 60 & 8 & 1.00 \\
\hline
\end{tabular}

\section{Results and discussion}

\subsection{Case study: IGC corrosion of single GB}

Fig. 9 shows the comparison between $v_{z, \operatorname{sim}}(\theta, \varphi=0)$ for each grid and $v_{z, t h}=\sin \theta$. In this particular $\varphi=0$ case, it appears clearly that the HCP and FCC-111 grids results compare better to $v_{z, t h}$ than those of the cubic one, which exhibit strong deviations from the isotropic $\sin \theta$ curve for $\varphi>30^{\circ}$, and remains equal to one for $\varphi>45^{\circ}$. Fig. 10 shows the comparison between $v_{z, \operatorname{sim}}\left(\theta=30^{\circ}, \varphi\right)$ and $v_{z, t h}=\sin \theta$ for each grid, as a function of $\varphi$ (then $v_{z, t h}$ is constant equal to 0.5 ). Here again we see that the cubic grid case exhibit stronger deviations from $v_{z, t h}$ than both of the two others. Moreover the different symmetry properties of the grids [26], that have been briefly discussed in paragraph 2 as far as rotations around the $z$ axis are concerned, are clearly highlighted in this figure. 


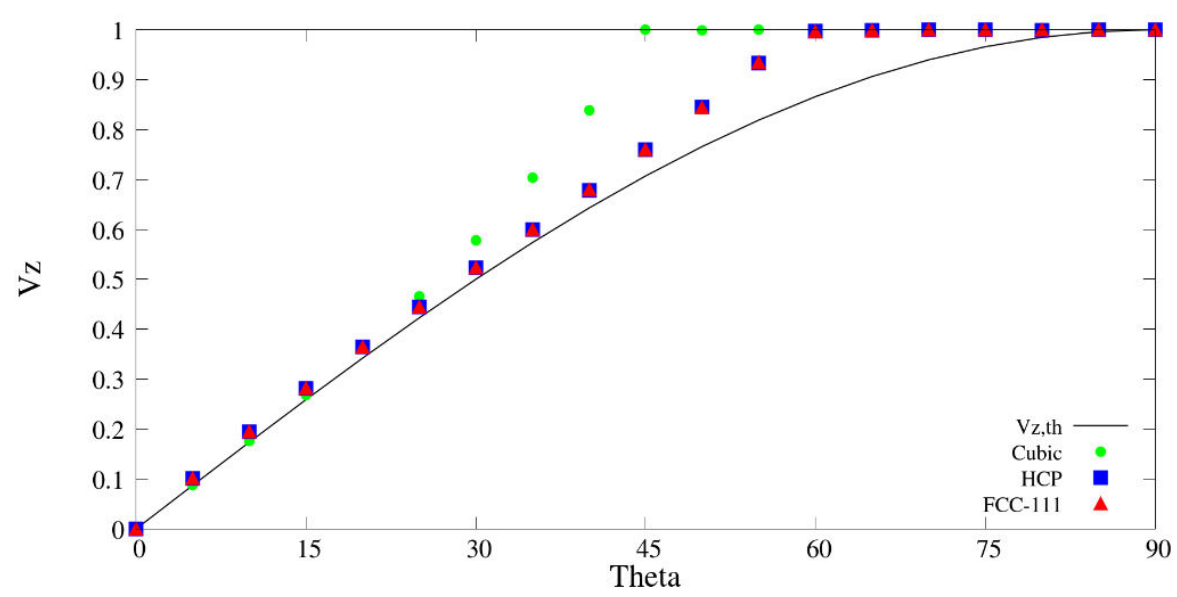

Figure 9 - Comparison between $v_{z, \operatorname{sim}}(\theta, \varphi=0)$ (greens discs for the cubic grid, blue squares for the HCP grid, red triangles for the FCC grid) and $v_{z, t h}=\sin \theta$ (black curve) as a function of $\theta$ (in degrees).

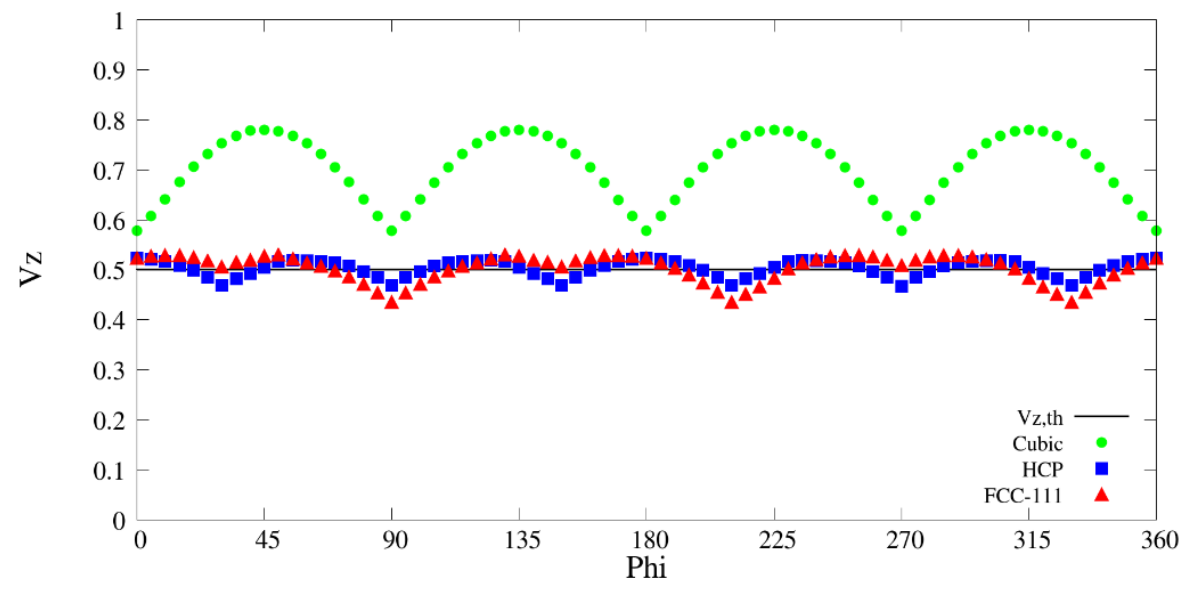

Figure 10 - Comparison between $v_{z, \operatorname{sim}}\left(\theta=30^{\circ}, \varphi\right)$ (greens discs for the cubic grid, blue squares for the HCP grid, red triangles for FCC grid) and $v_{z, t h}=\sin 30^{\circ}=0,5$ (horizontal black line) as a function of $\varphi$ (in degrees).

Let us now introduce (for each given grid) the following relative deviation

$\varepsilon(\theta, \varphi)=\frac{v_{z, s i m}(\theta, \varphi)-v_{z, t h}}{v_{z, t h}}$

We will note $<\varepsilon>_{\theta, \varphi}$ its arithmetic mean over the whole sample of $(\theta, \varphi)$ couples that were investigated (1296 in total), and $\sigma$ the standard deviation of the distribution $\varepsilon(\theta, \varphi)$.

The complete $\varepsilon(\theta, \varphi)$ angular screening results are summarized in Fig. 11, for the different grids, in terms of $\varepsilon(\theta, \varphi)$ (left column) and deviation $\varepsilon(\theta, \varphi)-<\varepsilon>_{\theta, \varphi}$ (right column).

Cubic grid results (Fig. 11, on top) clearly exhibit the expected 4-fold axial symmetry. GB corrosion seems to be slower when $\varphi=k * \frac{\pi}{2}, k=0,1,2, \ldots$ because SOL cells find more cells in their vertical path, while at corners $\left(\varphi=\frac{\pi}{4}+k * \frac{\pi}{2}, k=0,1,2, \ldots\right)$ the solution goes down more rapidly. This symmetric trend is clearly visible for $\theta<45^{\circ}$, then decreases when the plane tends to be vertical, where $v_{z, \operatorname{sim}}$ seems to be only $\theta$-dependent. The maximum relative deviation $\varepsilon_{\max }$ is reached for $\theta=40^{\circ}$ and is higher than $50 \%$. 
HCP grid results (Fig. 11, in the middle) present a symmetric pattern too. A complete $\varphi$-period is repeated 6 times, reflecting the 6-fold axial symmetry of the lattice [26]. The HCP grid results are globally far better than those of the cubic one.

As for the FCC-111 grid (Fig. 11, at the bottom), the expected 3-fold main axial symmetry is clearly visible. It shows to be almost as isotropic as HCP grid, with $\varepsilon_{\max }$ around $23 \%$. However, the $\left|\varepsilon_{\max }-\varepsilon_{\min }\right|$ difference increase is up to $36 \%$, that is more than in the HCP grid case.

A summary of the outcomes of the $(\theta, \varphi)$ screening is given in Table 3 , in terms of $\varepsilon_{\max }\left|\varepsilon_{\max }-\varepsilon_{\min }\right|,<\varepsilon>_{\theta, \varphi}$ and $\sigma$.
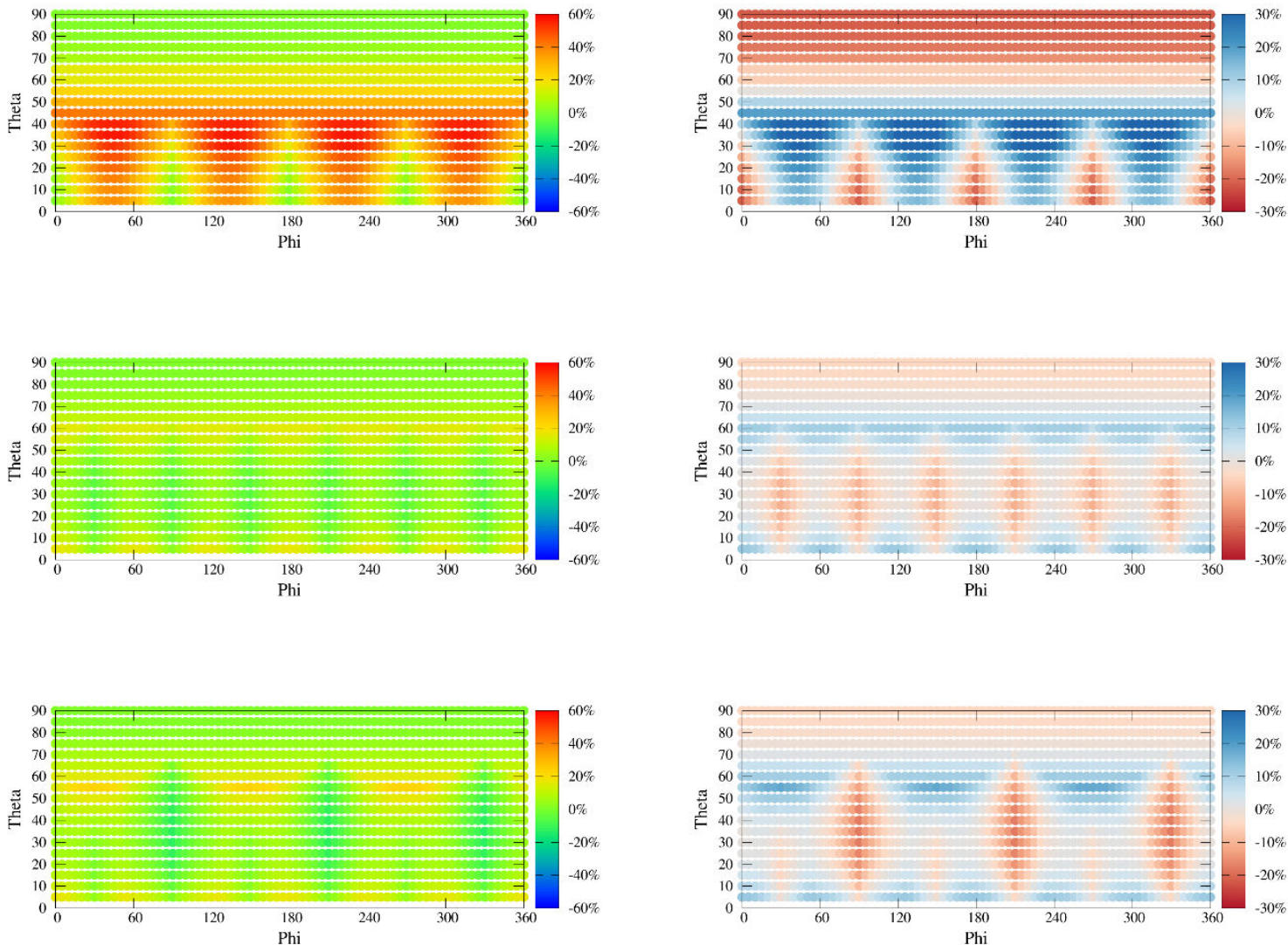

Figure 11 - Simulated GB corrosion rate relative deviation to isotropicity $(\varepsilon(\vartheta, \varphi)$, left column), and its deviation to its own mean $\left(\varepsilon(\vartheta, \varphi)-\langle\varepsilon\rangle_{\vartheta, \varphi}\right.$, right column), for the cubic (top), HCP (middle) and FCC-111 (bottom) grids.

Table 3 - Summary table of properties of the relative deviation $\varepsilon(\theta, \varphi)$

\begin{tabular}{|c|c|c|c|}
\hline & Cubic & HCP & FCC-111 \\
\hline$\varepsilon_{\max }$ & $56.7 \%$ & $16.7 \%$ & $22.9 \%$ \\
\hline$\left|\varepsilon_{\max }-\varepsilon_{\min }\right|$ & $56.7 \%$ & $22.9 \%$ & $36.2 \%$ \\
\hline$<\varepsilon>_{\theta, \varphi}$ & $22.0 \%$ & $4.8 \%$ & $4.7 \%$ \\
\hline$\sigma$ & $16.7 \%$ & $5.0 \%$ & $6.42 \%$ \\
\hline
\end{tabular}


Finally, HCP lattice showed to be the best in terms of relative deviation to isotropicity $\varepsilon(\theta, \varphi)$. In particular it gave the lowest $\left|\varepsilon_{\max }-\varepsilon_{\min }\right|$ difference and the lowest standard deviation.

To highlight the consequences of these results in a case which is more representative of a real material as a whole, we now present a second study where we simulate the IGC corrosion of SS samples that are generated by a Voronoï tessellation (like the one presented in Fig.1), with both cubic and HCP grids.

\subsection{Case study: IGC corrosion in a Voronoï}

The Voronoï tessellation is a useful model to build numerically a granular material microstructure that is made of convex grains [13]. We used it to build the initial conditions $(i=0)$ of the grids, that is made of IGN cells located at the grain boundaries (in red in Fig.1) and GN cells representing the convex grains (in brown in Fig.1). Simulations were performed on ( $N x=1536, N y=1536, N z=512)$ grids, both for the cubic and HCP cases. In the initial state of the grid, the two uppermost ( $z=N z-1$ and $z=N z$ ) layers consist of SOL cells (in blue in Fig.1), so that the corrosion progresses downwards, in the direction of decreasing z, as in the previous paragraph. The average characteristic width (or diameter) $d_{G}$ of the grains is set to around 215 cells (it is linked to the number of initial "seeds" cells, around 100, that are randomly chosen to initiate the grain growth phase in the Voronoï algorithm).

We present and compare the IGC cubic and HCP results through the evolution of the mass loss, to use a term commonly used in the experimental field. In real life experiments, this mass loss is the difference between the initial mass of the sample and its mass at time $t$, that is a monotonically increasing function of $t$ in the case of IGC [1]. In what follows we will consider that the mass loss is due either to the dissolution of IGN cells (which represent GB) or to grain detachments that occur each time a given grain is completely surrounded by SOL cells. In other words, if a group of GN cells representing a given grain is completely surrounded by SOL cells at iteration i, then we consider that all these GN cells (the grain) are detached from the sample, and it contributes to the mass loss. In this way, we see that the simulated mass loss $\Delta \mathrm{m}(\mathrm{i})$ at the i-th iteration is directly proportional to the difference between the initial total number of "material cells" $N_{\text {mat }}(i=0)=N_{I G N}(i=0)+N_{G N}(i=0)$ and its current value $N_{\text {mat }}(i)=N_{I G N}(i)+N_{G N}(i)$ (which represents IGN and GN cells that are not dissolved or detached from the bulk sample at iteration i). In order to eliminate the $\mathrm{N}_{\text {mat }}$ dependency to the lateral extension $\mathrm{Nx}{ }^{*} \mathrm{Ny}$ of the grid, we set

$\Delta m(i)=\frac{N_{\text {mat }}(i=0)-N_{\text {mat }}(i)}{N_{x y}} * A_{\text {grid }}$ 
where $A_{\text {grid }}$ is defined in paragraph 3 (see Table 1), $\mathrm{Nxy}=\mathrm{Nx} * \mathrm{Ny}$ is the total number of cells in a $\mathrm{z}$ plane of the grid, so that $\Delta \mathrm{m}(\mathrm{i})$ is finally equivalent to a mass loss per unit area of the sample exposed to the corrosive medium.

In order to facilitate further comparisons between numerical simulations and experimental results that often concern corrosion rates, we also computed a mass loss velocity $v_{\Delta m(i, j)}$, which is a sort of "parametric mean time-derivative" of $\Delta \mathrm{m}(\mathrm{i})$, that we defined by

$v_{\Delta m(i, j)}=\frac{\Delta m\left(i+\frac{j}{2}\right)-\Delta m\left(i-\frac{j}{2}\right)}{j}$

where $\mathrm{j}$ is a number that may serve as an adjustable parameter.

In Fig. 12 we compare $\Delta \mathrm{m}(\mathrm{i})$ and $v_{\Delta m(i, j)}$, with $\mathrm{j}=25$, for both the cubic and HCP grids.

As far as trends are considered, they are similar for both grids. We see that the mass loss velocity increases progressively as the number of iterations grows, before reaching the stationary "grain detachment" regime which is experimentally observed [1], where the corrosion velocity just oscillates around a steady state value. This regime is reached after about 300 iterations, which corresponds to the circumvention of about "one grain". Indeed, a given convex grain which mean "characteristic diameter" is $d_{G}$ can be circumvented with a line $L_{G}$ whose length is $(\pi / 2) d_{G}$. With $d_{G}=215$ cells, it gives $L_{G} \sim 340$ cells. Then the "grain detachment regime" is reached after about $L_{G}$ iterations. Note that from the moment this grain detachment regime is about to be reached (around 200/250 iterations), both the cubic and HCP corrosion velocities start to exhibit some "oscillations": these oscillations are due to "individual grains detachments" and are visible only because there is a finite number grains in each Voronoï. In other words, if we could handle numerically a much larger grid (containing a much larger number of grains), these oscillations would not appear so sharp, since detachments would appear in a quasi-continuous way. Of course, these "oscillations" are not synchronized from one simulation (involving a given Voronoï) to another. However, we clearly see that the cubic and HCP corrosion velocities differ from about $20 \%$, as expected from the results we presented in paragraph 4.1. Therefore, we confirm that HCP grid is the best choice to simulate IGC phenomena. A work is currently under progress to simulate IGC with this grid in a more representative way. As far as the time evolution of the mass loss is concerned, further quantitative comparisons with experiments will be achieved while introducing the grain dissolution phenomenon (which is present in real cases [1]) in the CA model. Time and space factors will be also set, to enable to relate the simulations number of iterations and cell size to the real time and space (or mass). 


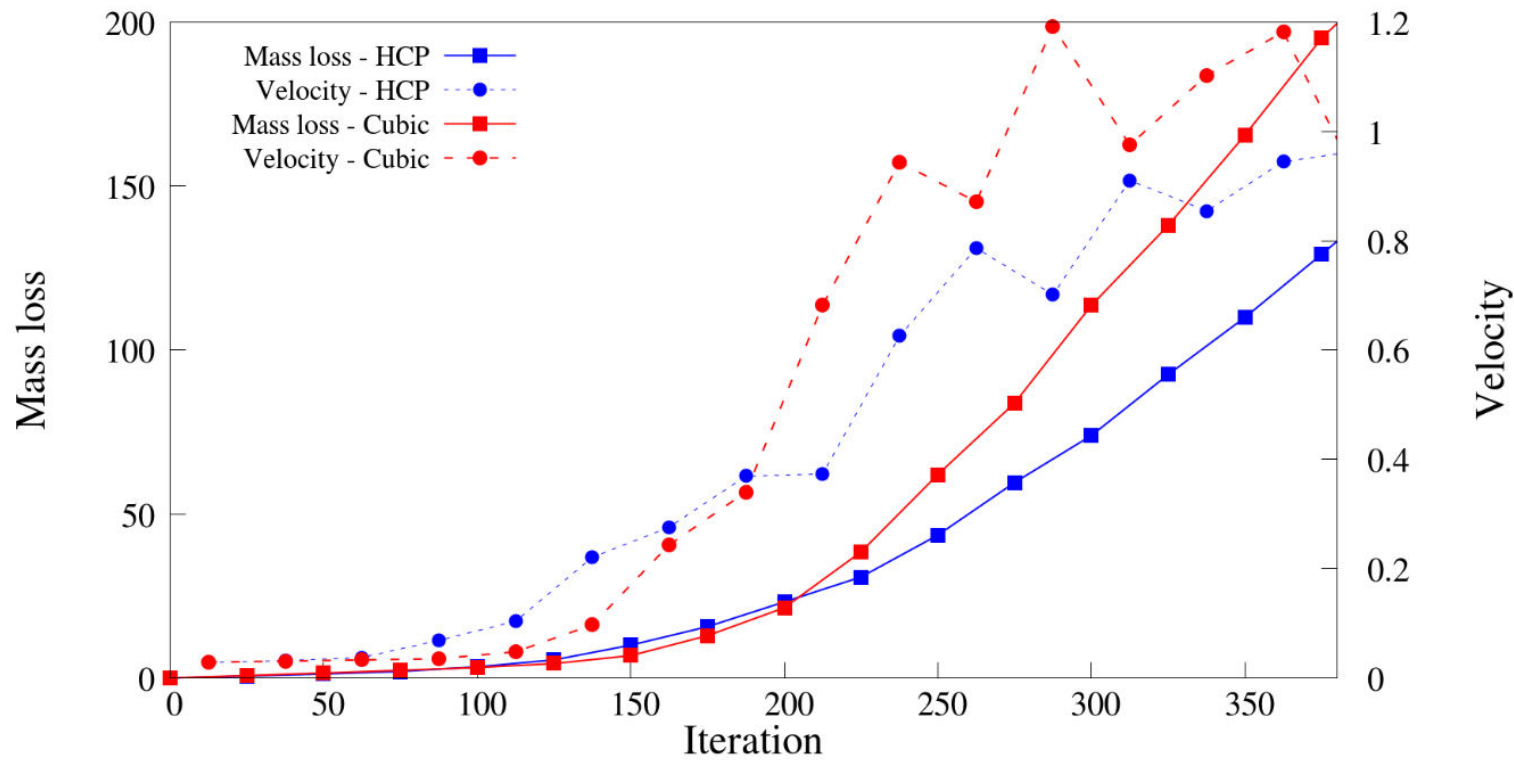

Figure 12 - Evolutions of the mass loss $\Delta \mathrm{m}(\mathrm{i})$ and the mass loss velocity $v_{\Delta m(i, j=25)}$ in the case of cubic (in red) and HCP (in blue) grids. The results are means over 8 simulations (that is 8 Voronoï diagrams) for each grid, with grains which mean characteristic sizes are around $d_{G} \sim 215$ cells.

\section{Conclusions}

In this paper, we modelled IGC through a 3D simple CA approach. We characterized three different types (cubic, HCP and FCC-111) of grids in terms of deviation to isotropy of the CA simulated GB corrosion rates, in order to find the best grid to be used for further IGC studies and detailed comparisons with experiments. Results showed that HCP grid is the most suitable to simulate IGC with a CA model, due to both its good intrinsic isotropicity properties and its relative simplicity of numerical implementation. The HCP lattice is here able to simulate IGC with a 5-10\% error margin, which can be considered as acceptable. The results we presented refer to the algorithm we chose to simulate IGC: a different algorithm could bring to new results.

\section{Acknowledgements}

The authors thank the CEA and the National Graduate School of Chemistry in Paris for supporting this work.

\section{References}

[1] B. Gwinner et al., "Towards a reliable determination of the intergranular corrosion rate of austenitic stainless steel in oxidizing media," Corrosion Science, vol. 107, pp. 60-75, Jun. 2016.

[2] W. D. Callister, Materials science and engineering: an introduction, 7th ed. New York: John Wiley \& Sons, 2007.

[3] B. Gwinner, J.-M. Borgard, E. Dumonteil, and A. Zoia, "A stereological approach for measuring the groove angles of intergranular corrosion," Corrosion Science, vol. 115, pp. 1-7, Feb. 2017.

[4] A. Emery "Intergranular corrosion mechanism of stainless steel (304L, Uranus 65) in nitric media" PhD thesis dissertation (2020). 
[5] M.L.C. Lim, R. G. Kelly, and J. R. Scully, "Overview of Intergranular Corrosion Mechanisms, Phenomenological Observations, and Modeling of AA5083," Corrosion, vol. 72, no. 2, pp. 198-220, Feb. 2016.

[6] S. Sarkar, J. E. Warner, W. Aquino, and M. D. Grigoriu, "Stochastic reduced order models for uncertainty quantification of intergranular corrosion rates," Corrosion Science, vol. 80, pp. 257-268, Mar. 2014.

[7] S. Zhao, D. A. Wolfe, T.-S. Huang, and G. S. Frankel, "Generalized model for IGC growth in aluminum alloys," Journal of Statistical Planning and Inference, vol. 137, no. 7, pp. 2405-2412, Jul. 2007.

[8] M. L. C. Lim, R. Matthews, M. Oja, R. Tryon, R. G. Kelly, and J. R. Scully, "Model to predict intergranular corrosion propagation in three dimensions in AA5083-H131," Materials \& Design, vol. 96, pp. 131-142, Apr. 2016.

[9] Ł. Bartosik, J. Stafiej, and D. Di Caprio, "Cellular automata model of anodization," Journal of Computational Science, vol. 11, pp. 309-316, Nov. 2015.

[10] C. F. Pérez-Brokate, D. di Caprio, É. Mahé, D. Féron, and J. de Lamare, "Cyclic voltammetry simulations with cellular automata," Journal of Computational Science, vol. 11, pp. 269-278, Nov. 2015.

[11] P. Van der Weeën, A. M. Zimer, E. C. Pereira, L. H. Mascaro, O. M. Bruno, and B. De Baets, "Modeling pitting corrosion by means of a 3D discrete stochastic model," Corrosion Science, vol. 82, pp. 133144, May 2014.

[12] H. Chen, Y. Chen, and J. Zhang, "Cellular automaton modeling on the corrosion/oxidation mechanism of steel in liquid metal environment," Progress in Nuclear Energy, vol. 50, no. 2-6, pp. 587-593, 2008.

[13] M. C. Chen and Q. Q. Wen, "Simulation of Corrosion Process for Structure with the Cellular Automata Method," in IOP Conference Series: Materials Science and Engineering, 2017, vol. 216.

[14] O. O. Fatoba, R. Leiva-Garcia, S. V. Lishchuk, N. O. Larrosa, and R. Akid, "Simulation of stress-assisted localised corrosion using a cellular automaton finite element approach," Corrosion Science, vol. 137, pp. 83-97, Jun. 2018.

[15] X. Gu, J. Kang, and J. Zhu, "3D Cellular Automata-Based Numerical Simulation of Atmospheric Corrosion Process on Weathering Steel," Journal of Materials in Civil Engineering, vol. 30, no. 11, Nov. 2018.

[16] L. Lei, L. Xiaogang, D. Chaofang, X. Kui, and L. Lin, "Cellular automata modeling on pitting current transients," Electrochemistry Communications, vol. 11, no. 9, pp. 1826-1829, Sep. 2009.

[17] K. Jahns, K. Balinski, M. Landwehr, V. B. Trindade, J. Wubbelmann, and U. Krupp, "Modeling of Intergranular Oxidation by the Cellular Automata Approach," Oxidation of Metals, vol. 87, no. 3-4, SI, pp. 285-295, Apr. 2017.

[18] D. di Caprio, J. Stafiej, G. Luciano, and L. Arurault, "3D cellular automata simulations of intra and intergranular corrosion," Corrosion Science, vol. 112, pp. 438-450, Nov. 2016.

[19] A. Taleb and J. Stafiej, "Numerical simulation of the effect of grain size on corrosion processes: Surface roughness oscillation and cluster detachment," Corrosion Science, vol. 53, no. 8, pp. 25082513, 2011.

[20] F. Ueno, A. Komatsu, T. Igarashi, and M. Yamamoto, "Study of the effect of phosphorus concentration on intergranular corrosion of stainless steel in boiling nitric acid solution," Eurocorr Pisa (Italy), 2014.

[21] T. Igarashi, A. Komatsu, T. Motooka, F. Ueno, Y. Kaji, and M. Yamamoto, "Simulations of intergranular corrosion feature for stainless steel using cellular automata method," Zairyo to Kankyo/ Corrosion Engineering, vol. 63, no. 7, pp. 431-437, 2014.

[22] S. V. Lishchuk, R. Akid, K. Worden, and J. Michalski, "A cellular automaton model for predicting intergranular corrosion," Corrosion Science, vol. 53, no. 8, pp. 2518-2526, Aug. 2011.

[23] S. V. Lishchuk, R. Akid, and K. Worden, "A cellular automaton based model for predicting intergranular corrosion in aerospace alloys," Proc. EUROCORR-2008, Edinburgh, UK, 2008.

[24] L. Hernández Encinas, S. Hoya White, A. Martín del Rey, and G. Rodríguez Sánchez, "Modelling forest fire spread using hexagonal cellular automata," Applied Mathematical Modelling, vol. 31, no. 6, pp. 1213-1227, Jun. 2007.

[25] J. Von Neumann and A. W. Burks, Theory of self-reproducing automata. Urbana and London: University of Illinois Press, 1966.

[26] B. Laue, "Crystal Structures and Symmetry," 2017. 
